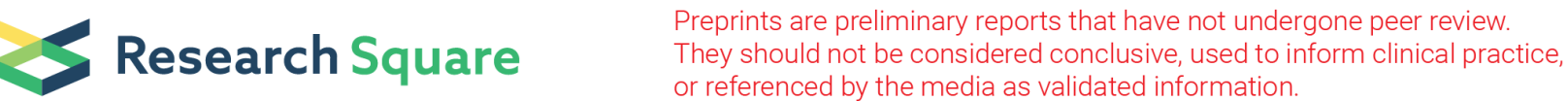

\section{Metabolic Heterogeneity of Brain Tumor Cells of Proneural and Mesenchymal Origin}

Corinna Seliger ( $\sim$ Corinna-Seliger-Behme@med.uni-heidelberg.de )

University Hospital Heidelberg: UniversitatsKlinikum Heidelberg

Verena Leidgens

University Hospital Regensburg

Anne-Louise Meyer

University Hospital Freiburg

Lisa Rauer

University Hospital Regensburg

Sylvia Moeckel

University Hospital Regensburg

Birgit Jachnik

University Hospital Regensburg

Judith Proske

University Hospital Regensburg

Katja Dettmer

University of Regensburg

Tanja Rothhammer-Hampl

University Hospital Regensburg: Universitatsklinikum Regensburg

Leon D. Kaulen

University Hospital Heidelberg: UniversitatsKlinikum Heidelberg

Markus J. Riemenschneider

University Hospital Regensburg: Universitatsklinikum Regensburg

Peter J. Oefner

University of Regensburg: Universitat Regensburg

Arabel Vollmann-Zwerenz

University Hospital Regensburg: Universitatsklinikum Regensburg

Marina Kreutz

University Hospital Regensburg: Universitatsklinikum Regensburg

Nils-Ole Schmidt

University Hospital Regensburg: Universitatsklinikum Regensburg

Marsha Merill

National Institute of Health

Martin Uhl 
University Hospital Erlangen

\section{Kathrin Renner}

University Hospital Regensburg: Universitatsklinikum Regensburg

\section{Martin Proescholdt}

University Hospital Regensburg: Universitatsklinikum Regensburg

\section{Peter Hau}

University Hospital Regensburg

\section{Research}

Keywords: glioma, metabolism, metformin

Posted Date: December 23rd, 2020

DOl: https://doi.org/10.21203/rs.3.rs-130871/v1

License: (c) (i) This work is licensed under a Creative Commons Attribution 4.0 International License. Read Full License 


\section{Abstract}

Background: Brain-tumor-initiating cells (BTICs) of proneural and mesenchymal origin contribute to the highly malignant phenotype of glioblastoma (GB) and resistance to current therapies.

Methods: BTICs of different subtypes representing tumor heterogeneity were challenged with OXPHOS (oxidative phosphorylation) inhibition to assess the differential effects of metabolic intervention on key resistance features.

Results: Whereas mesenchymal BTICs were more invasive, more glycolytic and less responsive to OXPHOS-inhibition, proneural BTICs were less invasive, catabolized glucose more via the pentose phosphate pathways, and responded better to OXPHOS inhibition.

Conclusion: Targeting glycolysis may be a promising approach to inhibit highly invasive tumor cells of mesenchymal origin, whereas proneural cells are more responsive to OXPHOS inhibition. Future clinical trials exploring metabolic interventions should account for metabolic heterogeneity of brain tumors to overcome resistance to current treatments.

\section{Background}

Glioblastoma (GB) is the most common and malignant primary brain tumor. Median overall survival of patients with GB ranges from 15 to 21 months $(1,2)$. Resistance to current therapy is thought at least in part to be driven by brain tumor initiating cells (BTICs) (3). BTICs are able to self-renew and to differentiate into different cell lineages (3). GBs are characterized by a significant inter- and intratumoral heterogeneity (4). They can be mainly classified into a classical, proneural and mesenchymal subtype according to their molecular profiles $(5,6)$. Mesenchymal BTICs are potentially more invasive than proneural BTICs (7). So far, the reasons for a differential invasiveness of specific tumor cells have not been understood conclusively. As invading tumor cells require significant amounts of energy (8) and use extracellular acidification and proteolytic degradation for their infiltration of healthy brain (9), we hypothesized that tumor metabolism may play a key role in their differential functional behavior.

Several metabolic interventions are currently explored in clinical trials (10). However, one major limitation of those approaches is insufficient accounting for metabolic heterogeneity. We performed several studies with approved drugs that target tumor metabolism including metformin (11-13). In line with differences in invasion capacity among BTIC subtypes, we observed different responses to metabolic drugs (11). Metformin was found to alter AMPK (adenosine monophosphate-activated protein kinase) and mTOR (mammalian target of rapamycin) signaling in tumor cells by inhibition of oxidative phosphorylation (14). It has thereby attracted great scientific interest as a potential anti-cancer drug. Derived from epidemiological data (15-19) and supported by in vitro and in vivo analyses (11-13, 20-22), metformin is currently in the focus of several ongoing clinical trials with glioma patients (NCT0143035, NCT02496741, NCT02149459, NCT02780024, NCT03243851, NCT03151772, NCT01528046, NCT02201381) mostly not accounting for metabolic heterogeneity. 
As we observed significant differences in invasion capacity among BTIC subtypes and variable responses to metabolic drugs, we hypothesized subtype-specific metabolic phenotypes that drive tumor cell malignancy. We propose that a better understanding of specific metabolic vulnerabilities of BTICs may spur the development of novel therapeutic strategies to overcome resistance to current therapies.

\section{Materials And Methods}

\section{Cellular models and functional assays}

\section{Tumor specimens and enrichment of BTICs}

BTICs were derived from resected, newly diagnosed human malignant gliomas as previously described (11). The sampling of tumor specimens and enrichment of BTICs was approved by the Ethics Committee of the University of Regensburg (No $\left.{ }^{\circ} 11-103-0182\right)$, and all patients gave written informed consent. BTICs were kept in DMEM low glucose media containing Epidermal Growth Factor and Fibroblast Growth Factor. For differentiation, growth factors were withdrawn and cells were exposed to fetal calf serum (FCS) for at least two weeks. Cells were incubated at $37^{\circ} \mathrm{C}, 5 \% \mathrm{CO}_{2}, 95 \%$ humidity in a standard tissue culture incubator.

\section{Cell proliferation and spheroid migration assays}

Proliferation was assessed using the CyQUANT ${ }^{\circledR}$ Direct Cell Proliferation Assay (Thermo Scientific, \#C35012) according to the manufacturers' protocol. The spheroid migration assays were performed as previously described (12).

\section{Organotypic brain slice cultures (OBSC)}

Lentivirally transduced BTICs were grown as spheroids in agarose coated 96 -well plates $(10,000$ cells/well) 48h prior to implantation. BTICs were lentivirally transduced using U57 pHR SFFV GFP (BTIC10/-12/-13) and pLenti-H1-(shRNA-Neg-control)-Rsv(RFP-Bsd) plasmids (BTIC-7/-8/-13). OBSC were prepared according to Gogolla et al.(23) with individual modifications as described (12).

\section{Treatment of BTICs with metformin}

Metformin hydrochloride (Sigma-Aldrich \#PHR1084) was dissolved in DMEM low glucose (5.5 mM) medium and used at indicated doses.

\section{RNA and protein-based methods}

\section{Polymerase Chain Reaction (PCR) and Quantitative Real Time PCR (qRT-PCR)}

RNA isolation and subsequent qRT-PCR isolation were performed as previously described (12). Primer sequences are provided in Suppl. Table 1. 


\section{Microarray analysis, Clustering and gene set enrichment analysis}

Microarray analysis was performed as described elsewhere.(24) Computational analysis was performed using R and Bioconductor (http://www.bioconductor.org). Genes differentially expressed between mesenchymal and proneural BTICs were identified using the package Limma (25). We computed a ranked gene list based on logarithmic fold change expression and applied the GSEA Preranked tool of GSEA v2.2.2 (www.broadinstitute.org/gsea). Gene set collections C2, C5 and C6 from the MSigDB (www.broadinstitute.org/msigdb) were used within GSEA. Enriched gene sets with an absolute normalized enrichment score $>2$ and a maximum false discovery rate (FDR) $<0.25 \%$ were considered for further evaluation.

\section{TCGA analysis}

mRNA expression of MCT1, MCT4 and isoforms of hexokinase, glucose-6-phosphate dehydrogenase and phosphofructokinase, was analyzed using the glioblastoma dataset available at the Cancer Genome Atlas (TCGA) database (26).

\section{Western Blot}

To investigate protein levels of (p)mTOR, (p)AMPK, (p)STAT3, LC3I/II, Caspase 3 and GAPDH, cells were harvested and lysed with RIPA buffer and subjected to Western blotting using standard protocols. Antibodies are listed in Suppl. Table 1.

\section{Metabolic assays}

\section{Enzyme activity assays}

Activity of hexokinase, phosphofructokinase and glucose-6-phosphate dehydrogenase was quantified using enzymatic assays provided by Abcam (\#ab136957) according to the manufacturer's recommendations.

\section{PreSens Technology}

Cellular oxygen consumption was determined non-invasively under culture conditions with PreSens technology (PreSens Precision Sensing $\mathrm{GmbH}$ ) as described (13).

\section{High-resolution respirometry}

To verify PreSens results on cellular oxygen consumption, activity of the respiratory system was analyzed in a two-channel titration injection respirometer (Oxygraph-2k; Oroboros Instruments, Innsbruck, Austria) at $37^{\circ} \mathrm{C}$. Detailed protocols are given in Suppl. Material 1. 
For quantitative analysis of intracellular metabolites, cells were collected and stored at $-80^{\circ} \mathrm{C}$. For further preparation, an internal standard solution containing stable isotope labeled analogs of the analytes was added. Samples were analysed by GC-MS after two-step derivatization using methoximation/silylation (27). For measurement of extracellular metabolites, a $10-\mu \mathrm{L}$ aliquot of the cell culture supernatant was dried and subjected to GC-MS analysis.

Quantification was achieved based on multi-point calibration curves for each analyte using the corresponding stable isotope labeled analog as internal standard.

\section{Extracellular acidification rate analysis by Seahorse technology}

Tumor cells were seeded in 96-well microplates at 8,000 cells per well in $80 \mu \mathrm{L}$ growth medium. One day prior to the experiment, the sensor cartridge was placed into the utility wells containing Seahorse XF calibrant and the system was incubated overnight at $37^{\circ} \mathrm{C}$ in a non- $\mathrm{CO}_{2}$ incubator. The next day, cells were washed in assay-specific medium and placed in the non-CO2 incubator for 1 hour. The ports of the Seahorse cartridge were loaded with $20 \mu \mathrm{L}$ of $80 \mathrm{mM}$ glucose, $22 \mu \mathrm{L}$ of $9 \mu \mathrm{M}$ oligomycin, and $25 \mu \mathrm{L}$ of 1 M 2DG for the glycolysis stress test. After sensor calibration, assays were run as detailed in the manufacturer's manual by recording ECAR (extracellular acidification rate) and OCR (oxygen consumption rate). Metabolic parameters were obtained from the XF Wave software (Agilent/Seahorse Biosciences) and calculated using Microsoft Excel.

\section{${ }^{13} \mathrm{C}-$ Glucose isotope tracing}

For stable isotope tracing experiments, cells were harvested and extracts prepared as described above without the addition of internal standards. We used $\left[{ }^{13} \mathrm{C}_{2-} 1,2\right]$ glucose as tracer substrate and measured metabolites derived therefrom. From the GC-MS full scan data, extracted ion chromatograms based on the $m / z$ values of the individual isotopologues were used for data analysis. Stable isotope tracing data were corrected for natural abundance of ${ }^{13} \mathrm{C}$ and tracer impurity using IsoCorrectoR (28).

The use of $\left[{ }^{13} \mathrm{C}_{2-1} 1,2\right]$ glucose as tracer substrate allows to determine the fractions of glucose catabolized via glycolysis and the pentose phosphate pathway. Single-labeled $\left[{ }^{13} \mathrm{C}_{1}-3\right]$ pyruvate and $\left[{ }^{13} \mathrm{C}_{1}-3\right]$ lactate $(m+1)$, following correction for natural abundance of ${ }^{13} \mathrm{C}$, provide an estimate of the fraction of glucose catabolized via the PPP, whereas double-labeled $\left[{ }^{13} \mathrm{C}_{2}-2,3\right]$ pyruvate and $\left[{ }^{13} \mathrm{C}_{2}-2,3\right]$ lactate $(\mathrm{m}+2)$ derive

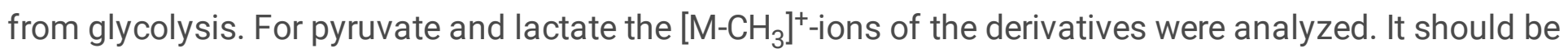
noted that positional information for ${ }^{13} \mathrm{C}$ incorporation is not achieved. Double-labeled pyruvate and lactate may alternatively derive from the PPP, but in that case a different labeling pattern is observed, namely $\left[{ }^{13} \mathrm{C}_{2}-1,3\right]$ pyruvate and $\left[{ }^{13} \mathrm{C}_{2}-1,3\right]$ lactate. Therefore, to unequivocally distinguish ${ }^{13} \mathrm{C}$ carbon flow between glycolysis and the PPP, we additionally analyzed a fragment of the lactate derivative containing only C2 to C3 $(\mathrm{m} / \mathrm{z} 117[\mathrm{~m}+0], 118[\mathrm{~m}+1], 119[\mathrm{~m}+2])$.

\section{Statistics}


All statistical analyses were performed using GraphPad Prism Version 7.05 (GraphPad Software, Inc. San Diego, USA) if not otherwise indicated.

We calculated a one-way ANOVA to compare the results (mean values and SDs) of controls and treated BTICs or between molecular subgroups. For molecular subgroups, we grouped the mean values of each proneural or mesenchymal BTIC or TC in the respective experiment. Assays were all performed in triplicate with two biological replicates. We used Tukey's post-hoc test to control for multiple comparisons. The level of significance was set at $p<0.05$ (asterisks indicate ${ }^{*} p<0.05{ }^{* *} p<0.01$, and $\star \star \star x<0.001)$. Western blots were repeated three times with at least two biological replicates and quantified using Image J, version 1.49.

When analyzing survival data in TCGA, we divided groups into "high" and "low" expression of the respective mRNA (i.e.MCT4 and MCT1) of GBs documented in TCGA using the median expression as cutoff (expression above versus below the median). Significance between groups was tested using the logrank (Mantel-Cox) test.

\section{Results}

\section{Mesenchymal BTICs were more invasive than proneural BTICs}

We analyzed 8 different primary BTIC lines and their respective differentiated counterparts (TCs) (Suppl. Table 2). Four BTIC lines each were classified as proneural (BTIC-7, BTIC-8, BTIC-17, BTIC-18) and mesenchymal (BTIC-10, BTIC-11, BTIC-12, BTIC-13) according to the Verhaak classification (5) based on microarray analyses. Classification into molecular subgroups was confirmed using next generation sequencing and expression of proneural and mesenchymal markers in immunocytochemistry.

Invasion of tumor cells into the surrounding healthy tissue is one major hallmark of glioblastoma. As we hypothesized that proneural and mesenchymal cells might differ in their invasiveness, we explored invasion of BTICs and TCs on organotypic brain tumor slices (OBSCs). Therefore, green-fluorescent mesenchymal and red-fluorescent proneural BTICs and TCs were monitored over 14 days under the fluorescent microscope. Mesenchymal BTICs and TCs were significantly more invasive than proneural BTICs and TCs (Fig. 1), with the maximum migratory distance per day being about 18-times higher in mesenchymal than proneural cells. Other basal cell characteristics including proliferation, migration and expression of signaling molecules did not differ significantly among the lines, when explored under lowglucose conditions (5.5 mM) and normoxia (Suppl. Fig. 1).

\section{Proneural BTICs were more responsive to OXPHOS inhibition}

Previous assays had already indicated significant heterogeneity between BTICs and TCs treated with metabolic agents (11). To explore this observation further, we comprehensively assessed the response of several BTICs and TCs to metformin, which was used as a model substance to inhibit OXPHOS. 
The effects of metformin on proliferation were explored using the CyQuant Direct Cell Proliferation Assay 48 and 96 hours after treatment with various concentrations of metformin. Treatment with metformin led to a dose- and time-dependent inhibition of proliferation among all investigated BTICs and TCs (Suppl. Fig. 2). Interestingly, proneural BTICs were significantly more responsive to metformin than mesenchymal BTICs and TCs, especially at lower concentrations of metformin (Fig. 2A).

Next, we evaluated, whether the observed differences in proliferation might be due to apoptosis, as demonstrated previously (29). In our model, $1 \mu \mathrm{M}$ staurosporine reliably induced apoptosis, as indicated by increased expression of cleaved caspase 3 in BTICs and TCs (Suppl. Fig. 3). In contrast, we did not observe a significant increase in caspase cleavage after treatment with increasing doses of metformin in most of the BTICs. We did observe a dose-dependent activation of AMPK and inhibition of MTOR and STAT3 (Fig. 2B and Suppl. Fig. 3), leading to increased autophagy (Suppl. Fig. 3L-S). To confirm results from Western Blot analyses, we used a vector containing the human LC3B gene fused to green fluorescent protein (GFP) to visualize autophagosome formation (exemplarily shown for BTIC-8, Suppl. Fig. 3T).

The impact of increasing doses of metformin on migration was investigated in spheroid migration assays over 16, 24, 40 and 48 hours. Whereas high-, but not low-dose metformin inhibited migration of most BTICs and TCs, one mesenchymal cell line (BTIC-13) did not change its migration behavior regardless of the dose of metformin (Suppl. Fig. 4). When comparing the group of proneural and mesenchymal BTICs, proneural BTICs were significantly more responsive to high-dose metformin (Fig. 2C). Early time points were examined to distinguish migration from proliferation. Treatment with metformin led to a dose-dependent inhibition of cellular oxygen consumption with different basal oxygen consumption of BTIC-18 (Fig. 2D) and TC-18 (Fig. 2E.)

\section{Oxygen consumption did not correlate with molecular subtype}

We investigated routine and FCCP stimulated cellular respiration using high-resolution respirometry. In line with published data, metformin severely inhibited complex-I-dependent-respiration (Fig. 3A), leading to comparable effects of $0.5 \mu \mathrm{M}$ rotenone.

BTIC-18, which had shown high sensitivity to metformin in proliferation assays, respired markedly more than other BTICs at baseline and after use of the uncoupling agent FCCP. However, endogenous and FCCP stimulated respiration varied widely among other BTICs and TCs (Fig. 3B, C). Consequently, there were no significant general differences in cellular respiration between the group of proneural and mesenchymal BTICs and TCs (Fig. 3D, E).

Next, we hypothesized that differential response to OXPHOS inhibitors may be due to different abilities to use or activate glycolytic rescue mechanisms. Therefore, we explored extracellular metabolites. Interestingly, mesenchymal cells consumed far more glucose than proneural cells (Fig. 4A and 4B) and showed increased extracellular lactate levels. Treatment with metformin led to increased consumption of glucose and production of lactate. We also performed Seahorse analyses to gain deeper insights into 
extracellular acidification rates (ECAR) and glycolytic reserve capacity. The ECAR and the glycolytic reserve capacity were significantly higher in mesenchymal than proneural BTICs (Fig. 4C, D), which may however also be attributed to $\mathrm{CO}_{2}$ production by dehydrogenases.

\section{Mesenchymal BTICs showed increased expression of glycolytic genes}

To identify genetic differences underlying differential metabolic patterns of proneural and mesenchymal BTICs, we performed a gene set enrichment analysis based on the mRNA expression of 36 published BTICs including those used for the present functional and metabolic assays (30). Data are deposited at the gene expression omnibus (GEO) functional genomics data repository under the accession numbers GSE51305 and GSE76990. BTICs were segregated into proneural and mesenchymal BTICs according to the gene signatures published by Verhaak et al.(5) Interestingly, using hallmark analyses, we found the metabolic hallmark glycolysis to differ between proneural and mesenchymal BTICs (Fig. 5A), with mesenchymal BTICs showing a more glycolytic transcriptome. Significantly changed genes of the hallmark glycolysis are depicted in Suppl. Table 3, taking into consideration that microarray data may not necessarily reflect protein expression.

Among the differentially expressed glycolytic genes, we found monocarboxylate transporter 4 (MCT4), which is important for the outward transport of lactate to be one interesting candidate for future research. MCT4 mRNA expression was significantly higher in mesenchymal than proneural BTICs on both the mRNA (Suppl. Table 3) and protein level (Fig. 5B). Using data from the Cancer Genome Atlas (TCGA), we were able to confirm that MCT4 (Fig. 5C), but not MCT1 expression (Fig. 5D), was significantly higher in mesenchymal than proneural GBs. Intracellular lactate levels were higher in BTIC-8 and BTIC-18 than in BTIC-11 and BTIC-13 (Suppl. Fig. 5A), and reduced after treatment with metformin.

To determine if MCT4 plays a major role in mediating the response to metformin in proneural versus mesenchymal BTICs, we inhibited MCT4 in several BTICs and explored their response to metformin. Silencing of MCT4 with siRNA was feasible (Suppl. Fig. 5B-E), but did not increase the inhibitory effects of metformin on tumor cell proliferation and migration (Suppl. Fig. 5F, G), even when used after glutamine withdrawal or under hypoxia (data not shown). Based on our results, although MCT4 may play a role in the different metabolic preferences of proneural and mesenchymal BTICs, sensitivity to metformin is likely based on several different genetic and metabolic alterations. We hence investigated glycolysis in proneural and mesenchymal BTICs in more detail.

\section{Proneural BTICs redirected glucose to the PPP}

Considering that expression of glycolytic genes may not necessarily translate into increased glycolysis, we next investigated activity of the key glycolytic enzymes hexokinase, glucose-6-phosphate dehydrogenase, and phosphofructokinase. Their expression in TCGA varied according to enzyme isoforms (Suppl. Fig. 6). 
Mesenchymal BTICs showed increased activity of hexokinase and phosphofructokinase, whereas proneural BTICs showed increased activity of glucose-6-phosphate dehydrogenase (Fig. 6B-D, Suppl. Fig. $6 \mathrm{E}, \mathrm{K}, \mathrm{O})$. We suspected that proneural BTICs metabolized more glucose via the pentose-phosphate pathway (PPP). To prove this assumption, we performed pyruvate and lactate tracing using $\left[{ }^{13} \mathrm{C}_{2}{ }^{-}\right.$ 1,2]glucose as tracer substrate (Fig. 6A, E-J). Indeed, we were able to confirm increased flux through the PPP in BTIC-18 as indicated by the higher abundance of $\mathrm{m}+1$ isotopologues for pyruvate and lactate.

\section{Key findings could be related to clinical data}

To validate our in vitro data, we compared our results with survival data from glioma patients. First, high expression of MCT-4 (Suppl. Fig. 7A), but not MCT-1 (Suppl. Fig. 7B) translates into inferior patient survival in the TCGA cohort. Although not reaching statistical significance, there is a trend for improved survival among patients with high expression of G6PDH (Suppl. Fig. 7C).

Second, using a meta-analytic approach, we retrieved available data from published studies on clinical responses to metformin, overall and according to subgroups. There are several observational studies on metformin in glioma, several (mostly ongoing) clinical trials and few suggested treatment regimens outside of clinical trials (Suppl. Table 4). When retrieving data from observational studies relating use of metformin to overall (OS) or progression-free survival (PFS), there is a trend for prolonged OS and PFS of patients with GBM on metformin in some of the studies (Suppl. Fig. 7B, C). Interestingly, there is only one study on high-grade gliomas that stratified for WHO grade and found a significantly improved OS and PFS among patients with WHO grade III glioma using metformin.

\section{Discussion}

Glioblastomas are heterogeneous brain tumors (5). In our study, we observed different invasive patterns of proneural and mesenchymal BTICs that correlated with their differentiation and metabolic state. In summary, mesenchymal BTICs were more invasive, more glycolytic and less responsive to OXPHOS inhibition, whereas proneural BTICs were less invasive, showed an increased flux of glucose carbons via the pentose phosphate pathway and responded better to OXPHOS inhibition.

Similar to our data, in a recent study, Fayzullin et al. described a mesenchymal gene signature that was associated with increased invasion (7). Several candidate mechanisms were described, including CD44associated altered cell adhesion in mesenchymal cells (31). Increased invasion is also a common phenomenon in epithelial-to-mesenchymal transition (EMT) (32). Overexpression of genes derived from a mesenchymal signature is associated with inferior survival of glioma patients (33). Gliomas frequently shift towards the mesenchymal subclass at tumor recurrence (33). Therefore, the mesenchymal subtype is likely to drive invasion, one of the hallmarks of glioblastoma. It should be noted, however, that even within one tumor there may be a coexistence of molecular subtypes reflecting intra-tumoral heterogeneity (34). 
A link between invasion and glycolysis has been described earlier in a different context. In that model, miRNA-451 inhibits glioma cell proliferation and invasion by downregulating glucose transporter 1 (35). Treatment with the VEGF antibody bevacizumab led to increased tumor hypoxia, activated glycolysis and increased brain invasion of tumor cells in an intracranial GB xenograft model (36). Increased invasion is a consequence of activated glycolysis, as it creates an acidic environment that favors cancer compared to healthy cells (37). Lactate, the end product of glycolysis, is generated through lactate-dehydrogenase A (LDH-A), which was found to induce transcriptional regulation of matrix-metalloproteinase-2 (MMP-2) and integrin alpha(v)beta(3) in glioma cells, both facilitating migration.(38) Additionally, lactate is coordinated by TGF-beta2 (transforming growth factor), whose mRNA ranked as strongest regulated among the mesenchymal group in the hallmark glycolysis in our microarray analyses. TGF-beta2 is activated by thrombospondin, which is in turn sensitive to acidic $\mathrm{pH}$ and increased lactate levels (39).

Considering the easy clinical application and multilayer mode of action, we chose metformin as a model substance to study GB cell metabolism. Based on previous studies (21), we assumed that metformin's mode of action might lead to an increased response of proneural cells due to their common mutations within metabolic pathways (e.g. IDH)(5) - one of metformin's major targets.

Several studies already explored effects of metformin on glioma cells. They mainly focused on effects of high-dose metformin on different functions of glioma cells including proliferation, invasion, apoptosis, and differentiation of BTICs $(20,21,29)$. None of those studies investigated why some cells are more responsive to metformin than others, a heterogeneity that is specifically observed in BTICs from different origins.

For the first time, we demonstrated a specific inhibition of complex I of the respiratory chain by metformin in glioma cells using high-resolution respirometry. Further studies should compare the effects of metformin to known complex I inhibitors such as rotenone or piercidin also in unpermeabilized cells. In addition, as complex I can be bypassed by several metabolites, the effects of complex I inhibition on OXPHOS should be compared to additional ETC (electron transport chain) inhibitors such as myxothiazol. It should be noted, however, that although metformin inhibits complex I of the respiratory chain there are also several other targets of metformin (40).

Previous studies have not comprehensively explored effects of metformin on molecular subgroups of brain tumor cells. Our results from transcriptome analyses, Seahorse analyses, enzyme activity assays and analyses of extracellular metabolites found mesenchymal BTICs to be more glycolytic and less responsive to OXPHOS inhibition. It would be interesting to further check the effect of galactose in addition to glucose on cell invasion and viability, as this metabolite is catabolized at a slower rate through glycolysis. Also, further testing may explore the subtype of pyruvate kinase in different molecular groups, which influences the amount of ATP (adenosine triphosphate) derived from glycolysis. Microarray data from numerous BTICs showed that glycolytic genes were differentially expressed between proneural and mesenchymal BTICs. The lactate-transporter MCT4 and the glycolytic enzyme glucose-6-phosphate dehydrogenase (G6PDH) were two of the most interesting candidate genes and 
were hence further explored. Although a single knockdown of MCT4 with siRNA may not be functionally relevant for BTICs as they are able to compensate via MCT1, the investigation of MCT4 in glioma pathophysiology merits further research. Voss et. al (2017) described that MCT4 is enriched in areas of tumor hypoxia and that an inhibition of MCT4 binding to its chaperon basigin strongly inhibited tumor progression of glioblastoma stem cells (41). In line with our data, Lim et al. (2014) found an upregulation of MCT4 to correlate with an aggressive mesenchymal subset of GB (42). Our hypothesis was further corroborated when analyzing patient data from TCGA, where high expression of MCT4 was associated with inferior patient survival.

The activity of G6PDH was higher among proneural BTICs. Cells that use mitochondrial respiration and lipolysis produce significant amounts of reactive oxygen species (ROS). For compensation of ROS (reactive oxygen species), tumor cells need NADPH (nicotinamide adenine dinucleotide phosphate) for glutathione regeneration. The pentose phosphate pathway generates NADPH for the reductive biosynthesis of fatty acids and antioxidant defense, as well as ribose 5-phosphate for nucleotide biosynthesis (43).

Based on the results discussed before, targeting glycolysis may particularly affect invasive mesenchymal BTICs, whereas proneural BTICs are more responsive to OXPHOS inhibition. Interestingly, observational data revealed improved OS and PFS predominantly among patients with WHO grade III gliomas using metformin, indicating subgroups being more responsive to OXPHOS-inhibition. Lower grade gliomas and secondary glioblastomas more frequently express a proneural gene signature alongside with frequent mutations within $\operatorname{IDH}(5,44)$. Although based on limited experimental data (45), one clinical trial (NCT28601826) is exclusively exploring the effects of metformin on IDH-mutated solid tumors including glioma which will thereby challenge our observation of an increased susceptibility of BTICs of the proneural subtype to metformin.

\section{Conclusion}

We observed heterogeneous metabolic preferences of lowly and highly invasive BTICs, which also varied in their response to OXPHOS inhibition. Mesenchymal BTICs were more invasive and glycolytic, whereas proneural BTICs showed increased use of the pentose phosphate pathway and responded better to OXPHOS inhibition. Future clinical trials exploring metabolic interventions should account for metabolic heterogeneity of brain tumors. Interventions targeting only one metabolic pathway are prone to fail, unless the tumor is suspect to specific metabolic vulnerabilities.

\section{Abbreviations}




\begin{tabular}{|ll|}
\hline BTICs & Brain-tumor-initiating cells \\
\hline GB & Glioblastoma \\
\hline OXPHOS & Oxidative phosphorylation \\
\hline AMPK & Adenosine monophosphate-activated protein kinase \\
\hline mTOR & Mammalian target of rapamycin \\
\hline NCT & National Clinical Trial \\
\hline FCS & Fetal calf serum \\
\hline OBSC & Organotypic brain slice cultures \\
\hline RNA & Ribonucleic acid \\
\hline GFP & Green fluorescent protein \\
\hline RFP & Red fluorescent protein \\
\hline PCR & Polymerase Chain Reaction \\
\hline qRT-PCR & Quantitative Real Time PCR \\
\hline GSEA & Gene Set Enrichment Analysis \\
\hline FDR & False discovery rate \\
\hline TCGA & Cancer Genome Atlas \\
\hline STAT3 & Signal transducer and activator of transcription 3 \\
\hline LC3I/II & Light chain 3 \\
\hline RIPA & Radioimmunoprecipitation assay \\
\hline GAPDH & Glyceraldehyde 3-phosphate dehydrogenase \\
\hline GC-MS & Gas chromatography-mass spectrometry \\
\hline 2DG & 2-Deoxy-D-Glucose. \\
\hline ECAR & Extracellular acidification rate \\
\hline ECAR & Oxygen consumption rate \\
\hline PPP & Pentose phosphate pathway \\
\hline ANOVA & Analysis of variance \\
\hline SD & Standard deviation \\
\hline TC & Tumor cell \\
\hline Monocarboxylate transporter 1 \\
\hline TCT
\end{tabular}




\begin{tabular}{|ll|}
\hline BTICs & Brain-tumor-initiating cells \\
\hline MCT4 & Monocarboxylate transporter 4 \\
\hline FCCP & Trifluoromethoxy carbonylcyanide phenylhydrazone \\
\hline G6PDH & Glucose-6-phosphate dehydrogenase \\
\hline OS & Overall survival \\
\hline PFS & Progression-free survival \\
\hline CD44 & Cluster of differentiation \\
\hline EMT & Epithelial-to-mesenchymal transition \\
\hline miRNA & Micro RNA \\
\hline VEGF & Vascular Endothelial Growth Factor \\
\hline LDH-A & Lactate-dehydrogenase A \\
\hline MMP-2 & Matrix-metalloproteinase-2 \\
\hline TGF & Transforming growth factor \\
\hline pH & Potential of hydrogen \\
\hline IDH & Isocitrate dehydrogenase \\
\hline ETC & Electron transport chain \\
\hline ATP & Adenosine triphosphate \\
\hline ROS & Reactive oxygen species \\
\hline NADPH & Nicotinamide adenine dinucleotide phosphate \\
\hline
\end{tabular}

\section{Declarations}

\section{Ethics approval and consent to participate}

The sampling of tumor specimens and enrichment of BTICs was approved by the Ethics Committee of the University of Regensburg (No $\left.{ }^{\circ} 11-103-0182\right)$, and all patients gave written informed consent.

\section{Consent for publication}

All authors approved the manuscript and this submission.

\section{Availability of data and material}

Microarray data are deposited at the gene expression omnibus (GEO) functional genomics data repository under the accession numbers GSE51305 and GSE76990. 


\section{Competing interests}

V. Leidgens is now a paid employee of Novocure. All other authors declare no conflict of interest.

\section{Funding}

The study was funded by the German Research Foundation (KFO-262-P10 to CS) and the Wilhelm Sander Stiftung (2009.800.1/2 to PH). The founding sponsors had no role in the design of the manuscript, interpretation of data, writing of the manuscript, and in the decision to publish the results.

\section{Author contributions}

CS, VL, A-LM, LR, SM, BJ, JP, KD, TR-H, AV-Z and KR performed the experiments. CS, MJR, PJO, AV-Z, MK, $\mathrm{MU}, \mathrm{KR}, \mathrm{MP}$ and $\mathrm{PH}$ designed the study and/or supervised the experimental work. $\mathrm{N}-\mathrm{OS}$ and MM provided cell lines and specific assays. CS, VL, A-LM, LDK and PH wrote the manuscript and/or designed figures and tables. All authors read the manuscript and revised it for intellectual content.

\section{Acknowledgments}

We thank Ina Weig-Meckl, Eva-Maria Störr, Annette Lohmeier und Lisa Ellmann for excellent technical assistance. We also thank all members of the KFO 262 for a critical discussion and valuable ideas regarding our project.

The results shown here are in part based upon data generated by the TCGA Research Network: https://www.cancer.gov/tcga.

\section{References}

1. Stupp R, Taillibert S, Kanner A, Read W, Steinberg D, Lhermitte B, et al. Effect of Tumor-Treating Fields Plus Maintenance Temozolomide vs Maintenance Temozolomide Alone on Survival in Patients With Glioblastoma: A Randomized Clinical Trial. JAMA. 2017;318(23):2306-16.

2. Stupp R, Mason WP, van den Bent MJ, Weller M, Fisher B, Taphoorn MJ, et al. Radiotherapy plus concomitant and adjuvant temozolomide for glioblastoma. N Engl J Med. 2005;352(10):987-96.

3. Lathia JD, Mack SC, Mulkearns-Hubert EE, Valentim CL, Rich JN. Cancer stem cells in glioblastoma. Genes Dev. 2015;29(12):1203-17.

4. Patel AP, Tirosh I, Trombetta JJ, Shalek AK, Gillespie SM, Wakimoto H, et al. Single-cell RNA-seq highlights intratumoral heterogeneity in primary glioblastoma. Science (New York. 2014;344(6190):pp. 1396-401.

5. Verhaak RG, Hoadley KA, Purdom E, Wang V, Qi Y, Wilkerson MD, et al. Integrated genomic analysis identifies clinically relevant subtypes of glioblastoma characterized by abnormalities in PDGFRA, IDH1, EGFR, and NF1. Cancer cell. 2010;17(1):98-110. 
6. Wang Q, Hu B, Hu X, Kim H, Squatrito M, Scarpace L, et al. Tumor Evolution of Glioma-Intrinsic Gene Expression Subtypes Associates with Immunological Changes in the Microenvironment. Cancer cell. 2017;32(1):42-56. e6.

7. Fayzullin A, Sandberg CJ, Spreadbury M, Saberniak BM, Grieg Z, Skaga E, et al. Phenotypic and Expressional Heterogeneity in the Invasive Glioma Cells. Transl Oncol. 2019;12(1):122-33.

8. Zhang J, Goliwas KF, Wang W, Taufalele PV, Bordeleau F, Reinhart-King CA. Energetic regulation of coordinated leader-follower dynamics during collective invasion of breast cancer cells. Proc Natl Acad Sci U S A. 2019;116(16):7867-72.

9. Lai M, Vassallo I, Lanz B, Poitry-Yamate C, Hamou MF, Cudalbu C, et al. In vivo characterization of brain metabolism by (1) H MRS, (13) C MRS and (18) FDG PET reveals significant glucose oxidation of invasively growing glioma cells. International journal of cancer. 2018;143(1):127-38.

10. Seliger C, Hau P. Drug Repurposing of Metabolic Agents in Malignant Glioma. Int J Mol Sci. 2018;19(9).

11. Seliger C, Meyer AL, Renner K, Leidgens V, Moeckel S, Jachnik B, et al. Metformin inhibits proliferation and migration of glioblastoma cells independently of TGF-beta2. Cell cycle (Georgetown Tex. 2016;15(13):1755-66.

12. Leidgens V, Proske J, Rauer L, Moeckel S, Renner K, Bogdahn U, et al. Stattic and metformin inhibit brain tumor initiating cells by reducing STAT3-phosphorylation. Oncotarget. 2017;8(5):8250-63.

13. Gerthofer V, Kreutz M, Renner K, Jachnik B, Dettmer K, Oefner P, et al. Combined Modulation of Tumor Metabolism by Metformin and Diclofenac in Glioma. Int J Mol Sci. 2018;19(9).

14. Owen MR, Doran E, Halestrap AP. Evidence that metformin exerts its anti-diabetic effects through inhibition of complex 1 of the mitochondrial respiratory chain. The Biochemical journal. 2000;348 Pt 3:607 - 14 .

15. Adeberg S, Bernhardt D, Ben Harrabi S, Bostel T, Mohr A, Koelsche C, et al. Metformin influences progression in diabetic glioblastoma patients. Strahlenther Onkol. 2015;191(12):928-35.

16. Seliger C, Genbrugge E, Gorlia T, Chinot O, Stupp R, Nabors B, et al. Use of metformin and outcome of patients with newly diagnosed glioblastoma - pooled analysis. International journal of cancer. 2019.

17. Seliger C, Luber C, Gerken M, Schaertl J, Proescholdt M, Riemenschneider MJ, et al. Use of metformin and survival of patients with high-grade glioma. International journal of cancer. 2018.

18. Seliger C, Ricci C, Meier CR, Bodmer M, Jick SS, Bogdahn U, et al. Diabetes, use of antidiabetic drugs, and the risk of glioma. Neurooncology. 2016;18(3):340-9.

19. Welch MR, Grommes C. Retrospective analysis of the effects of steroid therapy and antidiabetic medication on survival in diabetic glioblastoma patients. CNS Oncol. 2013;2(3):237-46.

20. Sato A, Sunayama J, Okada M, Watanabe E, Seino S, Shibuya K, et al. Glioma-initiating cell elimination by metformin activation of FOXO3 via AMPK. Stem cells translational medicine. 2012;1(11):811-24. 
21. Wurth R, Pattarozzi A, Gatti M, Bajetto A, Corsaro A, Parodi A, et al. Metformin selectively affects human glioblastoma tumor-initiating cell viability: A role for metformin-induced inhibition of Akt. Cell cycle (Georgetown Tex. 2013;12(1):145-56.

22. Shen H, Yu M, Tsoli M, Chang C, Joshi S, Liu J, et al. Targeting reduced mitochondrial DNA quantity as a therapeutic approach in pediatric high-grade gliomas. Neurooncology. 2020;22(1):139-51.

23. Gogolla N, Galimberti I, DePaola V, Caroni P. Preparation of organotypic hippocampal slice cultures for long-term live imaging. Nat Protoc. 2006;1(3):1165-71.

24. Moeckel S, Meyer K, Leukel P, Heudorfer F, Seliger C, Stangl C, et al. Response-predictive gene expression profiling of glioma progenitor cells in vitro. PloS one. 2014;9(9):e108632.

25. Smyth GK, Michaud J, Scott HS. Use of within-array replicate spots for assessing differential expression in microarray experiments. Bioinformatics. 2005;21(9):2067-75.

26. Brennan CW, Verhaak RG, McKenna A, Campos B, Noushmehr H, Salama SR, et al. The somatic genomic landscape of glioblastoma. Cell. 2013;155(2):462-77.

27. Singer K, Dettmer K, Unger P, Schonhammer G, Renner K, Peter K, et al. Topical Diclofenac Reprograms Metabolism and Immune Cell Infiltration in Actinic Keratosis. Front Oncol. 2019;9:605.

28. Heinrich P, Kohler C, Ellmann L, Kuerner P, Spang R, Oefner PJ, et al. Correcting for natural isotope abundance and tracer impurity in MS-, MS/MS- and high-resolution-multiple-tracer-data from stable isotope labeling experiments with IsoCorrectoR. Sci Rep. 2018;8(1):17910.

29. Isakovic A, Harhaji L, Stevanovic D, Markovic Z, Sumarac-Dumanovic M, Starcevic V, et al. Dual antiglioma action of metformin: cell cycle arrest and mitochondria-dependent apoptosis. Cell Mol Life Sci. 2007;64(10):1290-302.

30. Moeckel S, Vollmann-Zwerenz A, Proescholdt M, Brawanski A, Riemenschneider MJ, Bogdahn U, et al. Validation Study: Response-Predictive Gene Expression Profiling of Glioma Progenitor Cells In Vitro. PloS one. 2016;11(3):e0151312.

31. Brown DV, Daniel PM, D'Abaco GM, Gogos A, Ng W, Morokoff AP, et al. Coexpression analysis of CD133 and CD44 identifies proneural and mesenchymal subtypes of glioblastoma multiforme. Oncotarget. 2015;6(8):6267-80.

32. Kalluri R. EMT: when epithelial cells decide to become mesenchymal-like cells. J Clin Invest. 2009;119(6):1417-9.

33. Phillips HS, Kharbanda S, Chen R, Forrest WF, Soriano RH, Wu TD, et al. Molecular subclasses of high-grade glioma predict prognosis, delineate a pattern of disease progression, and resemble stages in neurogenesis. Cancer cell. 2006;9(3):157-73.

34. Bergmann N, Delbridge C, Gempt J, Feuchtinger A, Walch A, Schirmer L, et al. The Intratumoral Heterogeneity Reflects the Intertumoral Subtypes of Glioblastoma Multiforme: A Regional Immunohistochemistry Analysis. Front Oncol. 2020;10:494.

35. Guo H, Nan Y, Zhen Y, Zhang Y, Guo L, Yu K, et al. miRNA-451 inhibits glioma cell proliferation and invasion by downregulating glucose transporter 1. Tumour Biol. 2016;37(10):13751-61. 
36. Keunen O, Johansson M, Oudin A, Sanzey M, Rahim SA, Fack F, et al. Anti-VEGF treatment reduces blood supply and increases tumor cell invasion in glioblastoma. Proc Natl Acad Sci U S A. 2011;108(9):3749-54.

37. Gatenby RA, Gillies RJ. Why do cancers have high aerobic glycolysis? Nat Rev Cancer. 2004;4(11):891-9.

38. Baumann F, Leukel P, Doerfelt A, Beier CP, Dettmer K, Oefner PJ, et al. Lactate promotes glioma migration by TGF-beta2-dependent regulation of matrix metalloproteinase-2. Neurooncology. 2009;11(4):368-80.

39. Seliger C, Leukel P, Moeckel S, Jachnik B, Lottaz C, Kreutz M, et al. Lactate-modulated induction of THBS-1 activates transforming growth factor (TGF)-beta2 and migration of glioma cells in vitro. PloS one. 2013;8(11):e78935.

40. Lei Y, Yi Y, Liu Y, Liu X, Keller ET, Qian CN, et al. Metformin targets multiple signaling pathways in cancer. Chin J Cancer. 2017;36(1):17.

41. Voss DM, Spina R, Carter DL, Lim KS, Jeffery CJ, Bar EE. Disruption of the monocarboxylate transporter-4-basigin interaction inhibits the hypoxic response, proliferation, and tumor progression. Sci Rep. 2017;7(1):4292.

42. Lim KS, Lim KJ, Price AC, Orr BA, Eberhart CG, Bar EE. Inhibition of monocarboxylate transporter-4 depletes stem-like glioblastoma cells and inhibits HIF transcriptional response in a lactateindependent manner. Oncogene. 2014;33(35):4433-41.

43. Jin L, Zhou Y. Crucial role of the pentose phosphate pathway in malignant tumors. Oncol Lett. 2019;17(5):4213-21.

44. Popova SN, Bergqvist M, Dimberg A, Edqvist PH, Ekman S, Hesselager G, et al. Subtyping of gliomas of various $\mathrm{WHO}$ grades by the application of immunohistochemistry. Histopathology. 2014;64(3):365-79.

45. Cuyas E, Fernandez-Arroyo S, Corominas-Faja B, Rodriguez-Gallego E, Bosch-Barrera J, MartinCastillo B, et al. Oncometabolic mutation IDH1 R132H confers a metformin-hypersensitive phenotype. Oncotarget. 2015;6(14):12279-96.

\section{Figures}




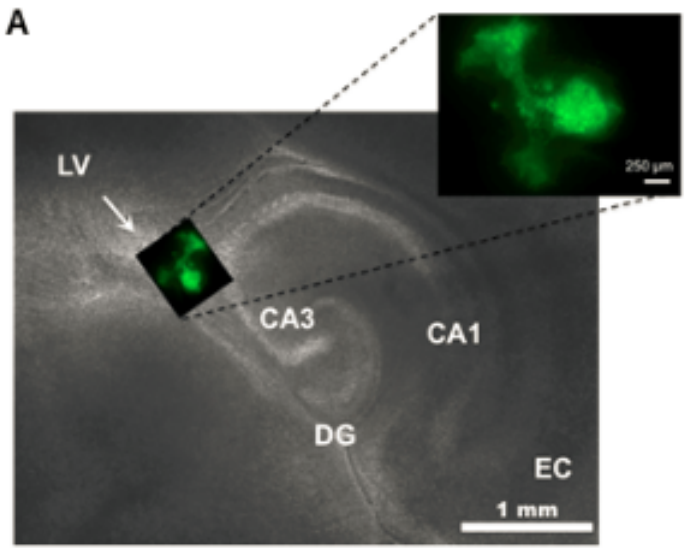

C Invasion of mesenchymal BTICs

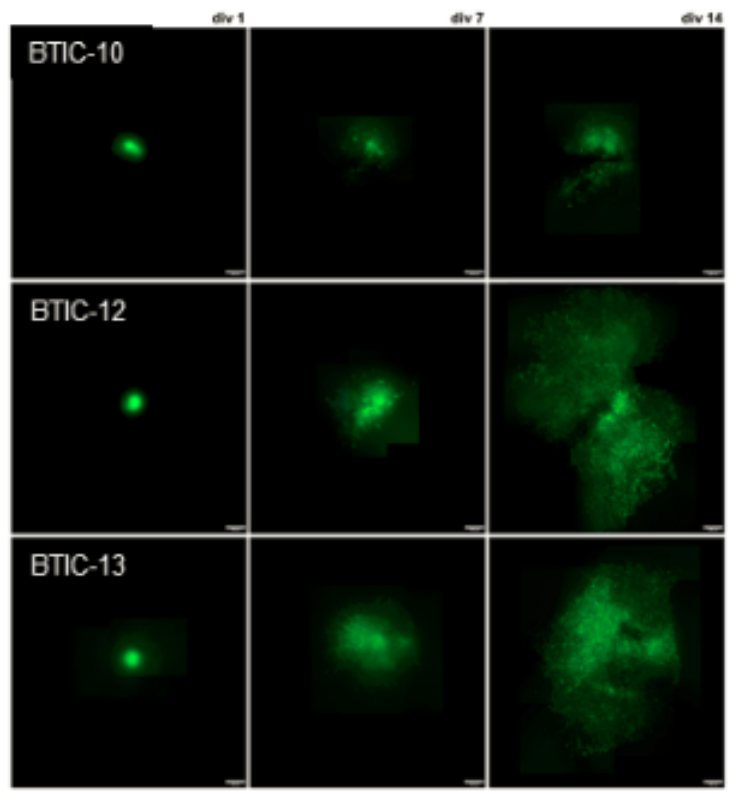

B

Area expansion over 14 days

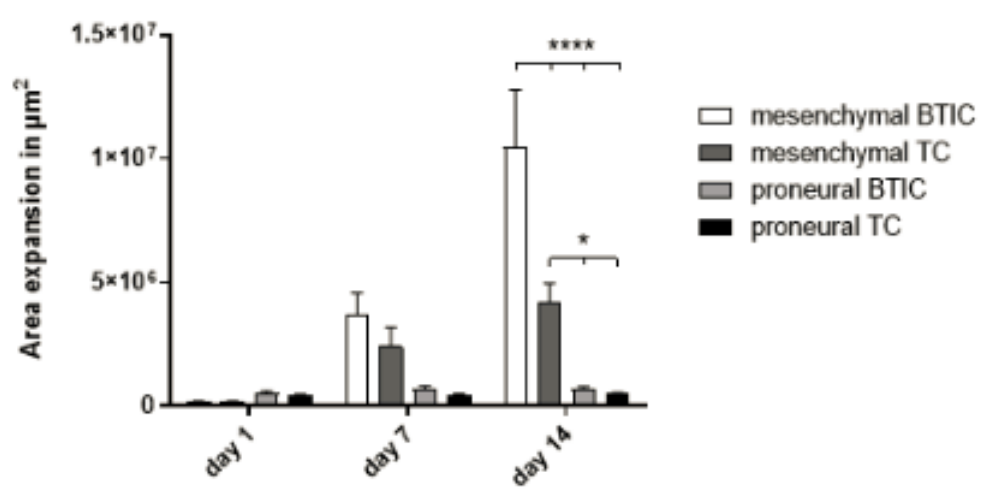

D Invasion of proneural BTICs

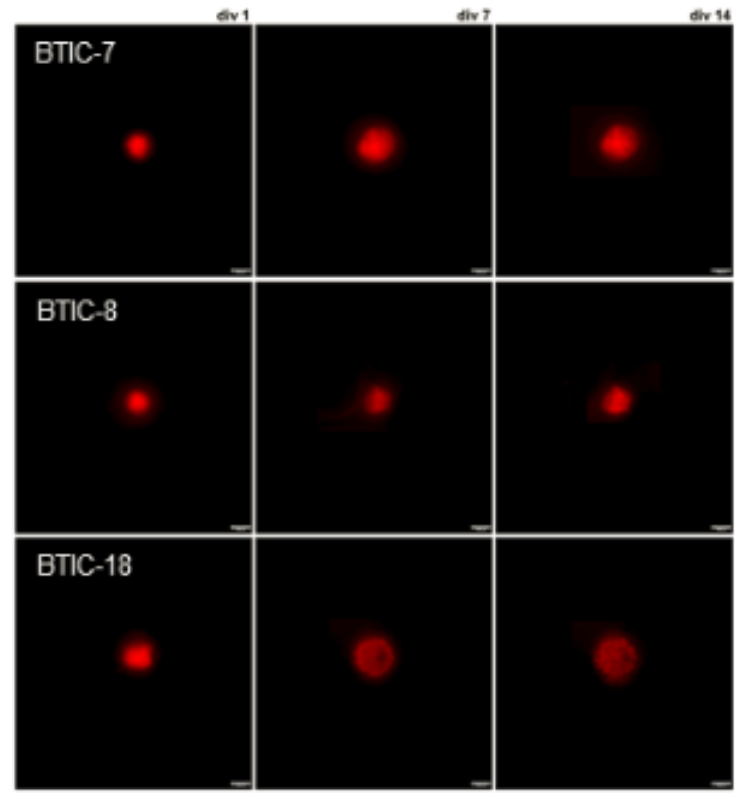

\section{Figure 1}

Invasion of BTICs and TCs. (A) Mesenchymal cells were lentivirally transduced with a U57 pHR SFFV GFP plasmid (green), while proneural cells were transduced with pLenti-H1-(shRNA-Neg-control)-Rsv(RFP-Bsd) (red). Two-day-old spheres of these transduced BTICs were transferred onto rat brain slices and inserted in the parahippocampal region. Invasion on organotypic brain slice cultures (OBSC) was monitored at 10x magnification under a fluorescent microscope on days 0,7 , and 14. (B) Mesenchymal BTICs and TCs (BTIC and TC-10, -12 and -13) were significantly more invasive than proneural BTICs and TCs (BTIC and TC-7, $-8,-18)$ on day 14 . Exemplary images of mesenchymal and proneural BTICs on OBSCs are depicted in (C) and (D); the scale bar indicates $250 \mu \mathrm{m}$. 

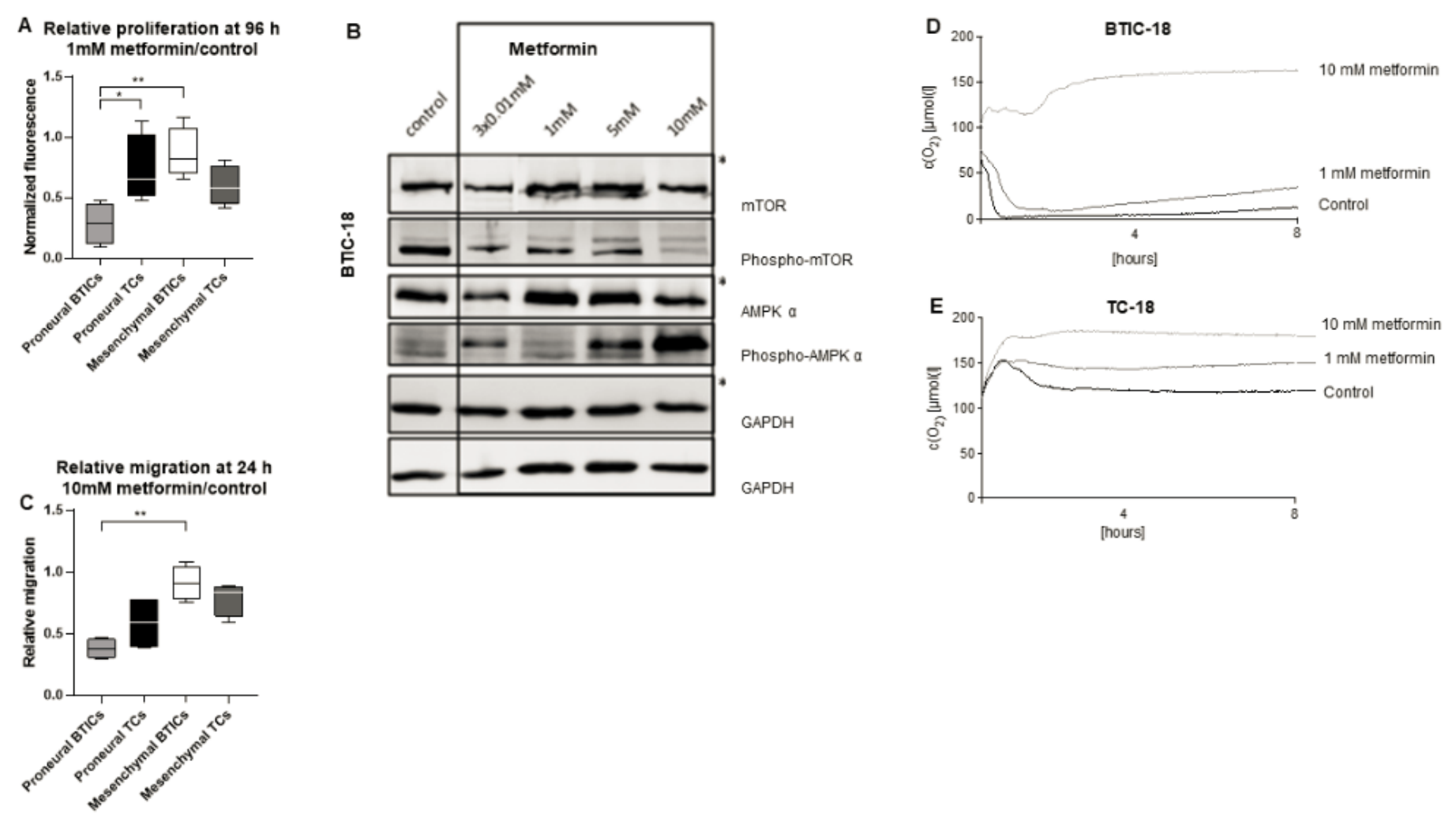

Figure 2

Effects of metformin on BTICs and TCs. (A) We compared proliferation of proneural (BTIC- and TC-7, -8, $-17,-18$ ) and mesenchymal (BTIC- and TC-10, -11, -12,-13) BTICs and TCs with or without $1 \mathrm{mM}$ metformin after $96 \mathrm{~h}$ of treatment. Proliferation was normalized to the 0-hour-value. (B) Increasing doses of metformin led to inhibition of mTOR and activation of the AMPK in Western Blot analyses after $48 \mathrm{~h}$ of treatment in BTIC-18. (C) Migration of proneural (BTIC- and TC-7, -8, -17, -18) and mesenchymal (BTICand TC-10, -11, -12, -13) BTICs and TCs with or without $10 \mathrm{mM}$ metformin was measured after $24 \mathrm{~h}$ of treatment. Migration was normalized to the 0-hour-value. Oxygen consumption over $8 \mathrm{~h}$ decreased under treatment with metformin in both (D) BTIC-18 and (E) TC-18, but levels of endogenous oxygen consumption varied significantly. 


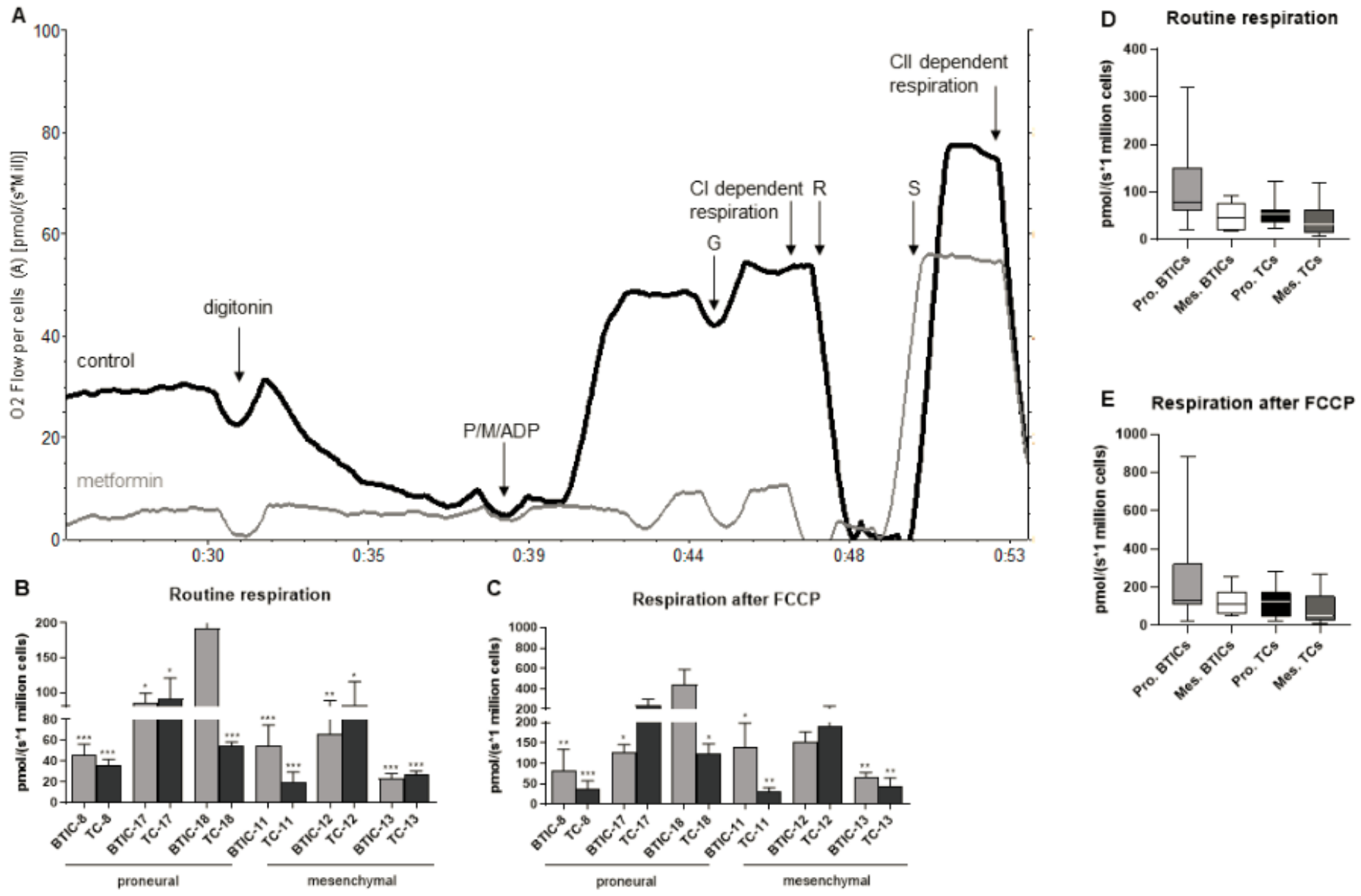

Figure 3

Respiration of BTICs and TCs. (A) Metformin (1 mM) inhibits complex I dependent respiration, but barely affects complex II dependent respiration. (B) Routine and (C) FCCP stimulated respiration varied widely between BTIC- and TC-8, $-17,-18,-11,-12$ and -13 cell lines. Grouping the lines according to their molecular subtype did not reveal significant results for (D) endogenous or (E) FCCP stimulated respiration. 
A

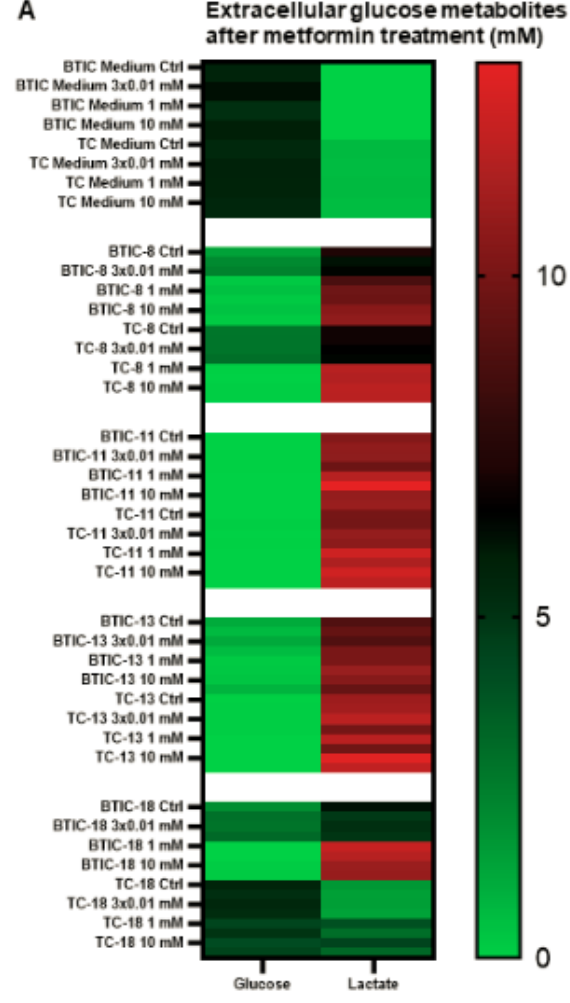

B

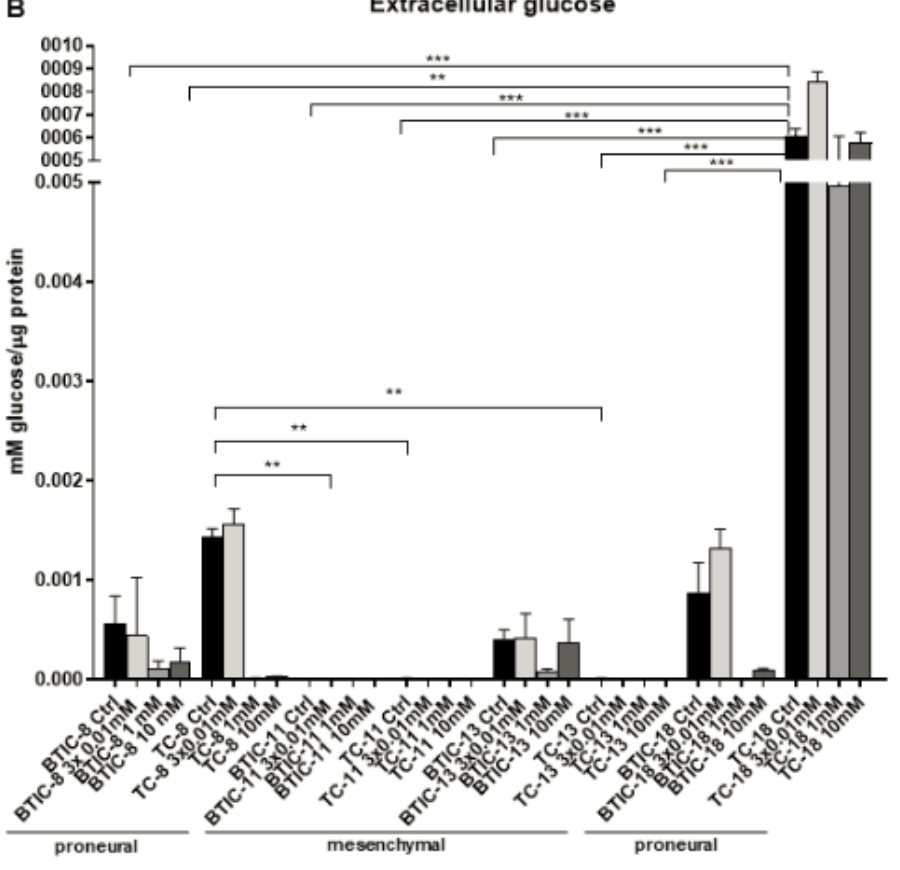

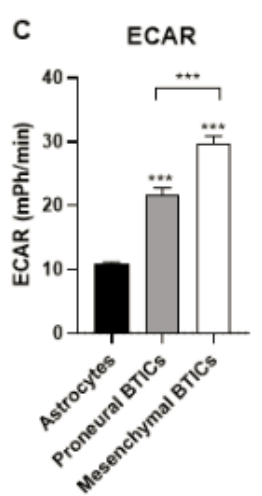

D Glycolytic reserve

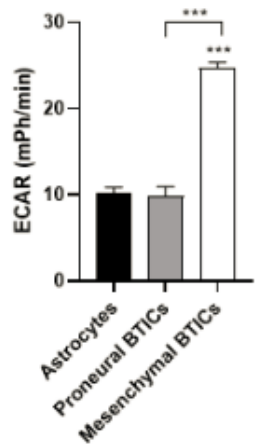

Figure 4

Metabolite expression and glycolytic activity. (A) We measured extracellular glucose and lactate after 48 $\mathrm{h}$ in BTIC- and TC-8, $-11,-13$ and -18 with or without treatment with $0.01 \mathrm{mM}$ metformin three times per day (tid), $1 \mathrm{~m} \mathrm{M}$ metformin and $10 \mathrm{mM}$ metformin using GC/MS. (B) Glucose values are also depicted per $\mu \mathrm{g}$ protein. (C) The extracellular acidification rate and (D) glycolytic reserve was significantly higher in mesenchymal than proneural BTICs in Seahorse Assays. 

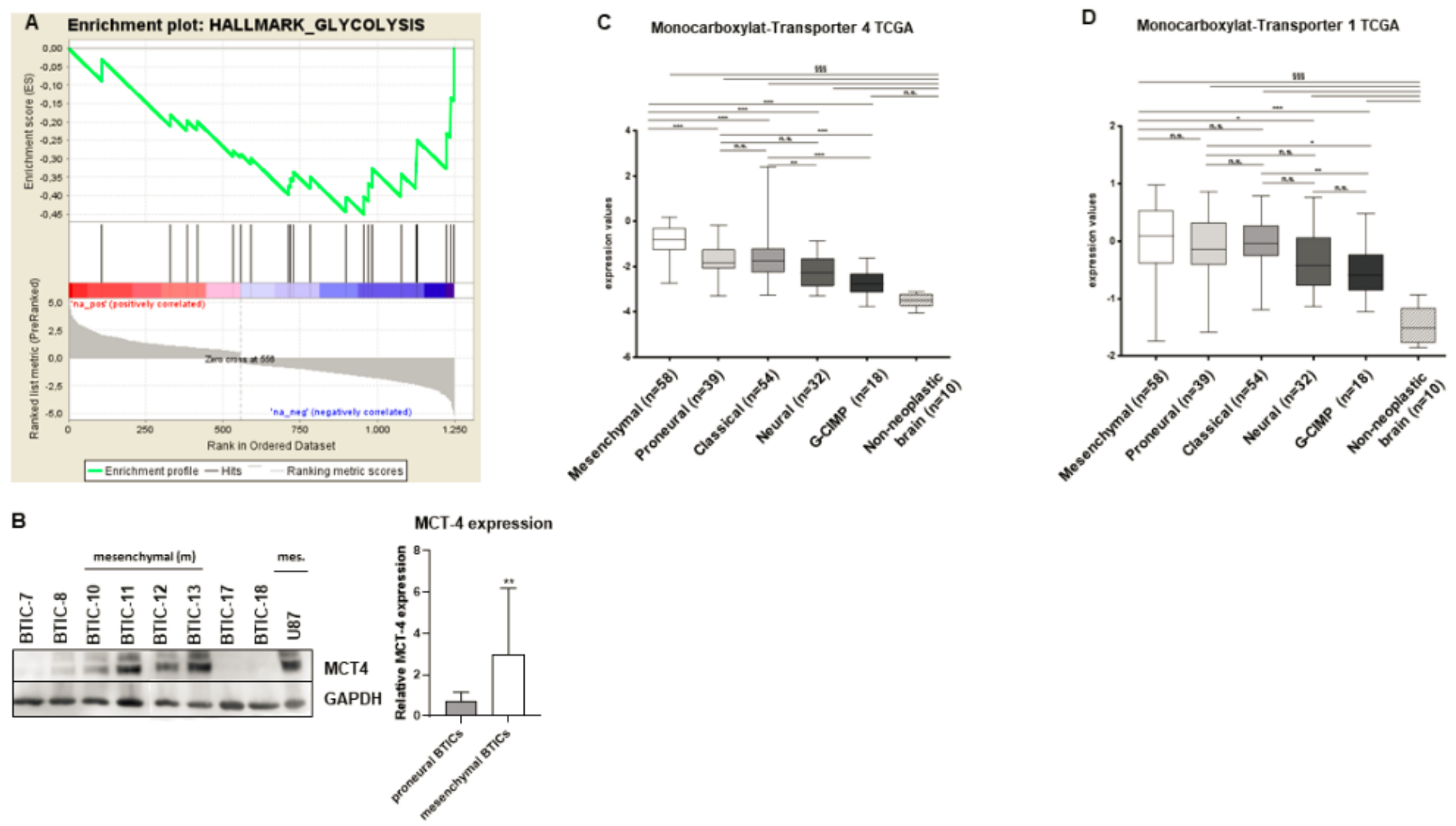

Figure 5

Hallmark analysis of mRNA-microarrays. (A) The enrichment plot indicates increased expression of glycolytic genes in mesenchymal BTICs (based on 36 lines including those used for this study). (B). We verified mRNA-microarray results by Western Blot. Thereby, an increased protein expression of MCT4 could be confirmed, which is shown in the exemplary blot and quantification of several biological and technical replicates. Array and expression results were validated using TCGA-data for (C) MCT4 and (D) MCT1. (E) MCT4 and (F) (MCT1) expression correlated with survival in patients documented in TCGA. 

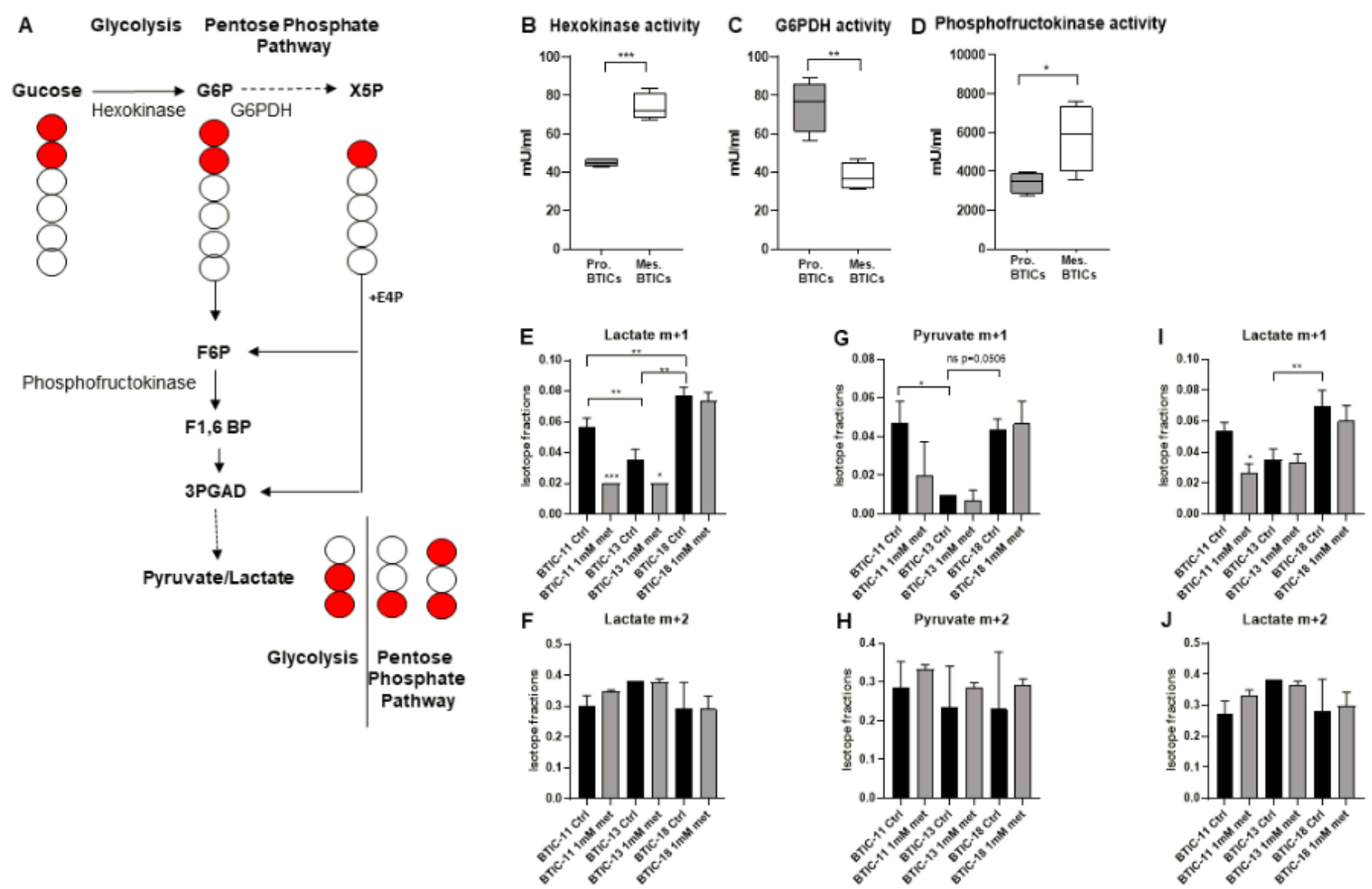

Figure 6

Activity of glycolytic enzymes and lactate tracing with [13C2-1,2]glucose. (A) Diagram depicting [13C21,2]glucose metabolism through glycolysis and the pentose phosphate pathway (PPP) and the resulting labeling patterns of key intermediates and lactate. Glycolysis will result in the formation of one molecule each of [13C2-2,3]lactate and [12C3]lactate from one molecule [13C2-1,2]glucose, while the PPP will convert three molecules of [13C2-1,2]glucose into one molecule each of [13C2-1,3]lactate and [13C13]lactate and three molecules of [12C3]lactate. The relative abundances of PPP-derived [13C1-3]lactate $($ lactate $\mathrm{m}+1)$ and glycolytic [13C2-2,3]lactate (lactate $\mathrm{m}+2)$ provide estimates of PPP and glycolytic activity, respectively. Key enzymes of glycolysis and the pentose-phosphate pathway are depicted in the graph. G6P=glucose 6-phosphate, X5P=xylulose 5-phosphate, G6PDH=glucose-6-phosphate dehydrogenase, $\mathrm{E} 4 \mathrm{P}=$ erythrose 4-phosphate, $\mathrm{F} 6 \mathrm{P}=$ fructose 6 -phosphate, $\mathrm{F} 1,6 \mathrm{BP}=$ fructose 1,6 bisphosphate, GAP = glyceraldehyde 3-phosphate. Red filled circles denote carbon 13 , black unfilled circles denote carbon 12. (B) Hexokinase activity was significantly lower in proneural (BTIC- and TC-7, -8, -17, -18) than mesenchymal (BTIC- and TC-10, -11, -12, -13) BTICs, while (C) the opposite applied to G6PDH activity. (D) Phosphofructosekinase activity corresponded to hexokinase activity. (E) The proneural BTIC18 contained significantly more PPP-derived [13C1-3] lactate (lactate $m+1$, lactate derivative containing only $\mathrm{C} 2$ to $\mathrm{C} 3$ ) than the mesenchymal BTICs 11 and 13. Treatment with $1 \mathrm{mM}$ metformin led to a significant drop in PPP-derived [13C1-3]lactate in BTIC-11 and BTIC-13. (F) The relative content of glycolytic [13C2-2,3]lactate (lactate $\mathrm{m}+2$, lactate derivative containing only $\mathrm{C} 2$ to $\mathrm{C} 3$ ) was increased in 
BTIC-13, albeit not significantly, as compared to BTIC-11 and -18. Treatment with $1 \mathrm{mM}$ metformin resulted in a relative, albeit not significant increase in [13C2-2,3]lactate in BTIC-11. Utilization of [13C21,2] glucose via the PPP leads to the formation of $(G)$ [13C1-3]pyruvate and $(H)$ [13C1-3]lactate, whose $\mathrm{m} / \mathrm{z}$ values increase by +1 unit in comparison to [12C3]pyruvate and [12C3-3]lactate. Utilization of [13C21,2]glucose via glycolysis results in double labelled $(\mathrm{m}+2)(\mathrm{I})$ [13C2-2,3]pyruvate and (J) [13C2-2,3]lactate. Hence, a higher fraction of $\mathrm{m}+1$ pyruvate or lactate isotopologues indicates increased PPP activity.

\section{Supplementary Files}

This is a list of supplementary files associated with this preprint. Click to download.

- SupplementaryMaterial.docx 